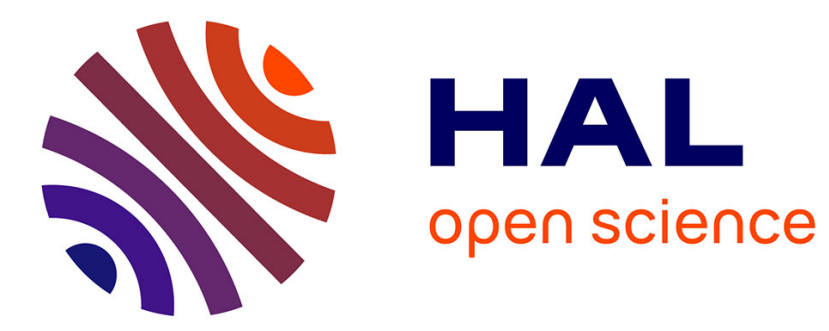

\title{
Imaginer les visiteuses : Scripts de genre et conception d'une exposition
}

\author{
Marion Coville
}

\section{To cite this version:}

Marion Coville. Imaginer les visiteuses : Scripts de genre et conception d'une exposition. Culture et Musées, 2017, 30, pp.73-90. 10.4000/culturemusees.1192 . halshs-02526110

\section{HAL Id: halshs-02526110 \\ https://shs.hal.science/halshs-02526110}

Submitted on 31 Mar 2020

HAL is a multi-disciplinary open access archive for the deposit and dissemination of scientific research documents, whether they are published or not. The documents may come from teaching and research institutions in France or abroad, or from public or private research centers.
L'archive ouverte pluridisciplinaire HAL, est destinée au dépôt et à la diffusion de documents scientifiques de niveau recherche, publiés ou non, émanant des établissements d'enseignement et de recherche français ou étrangers, des laboratoires publics ou privés. 


\section{IMAGINER LES VISITEUSES. \\ SCRIPTS DE GENRE ET CONCEPTION D'UNE EXPOSITION}

\section{Résumé
IMAGINER LES VISITEUSES. SCRIPTS DE GENRE ET CONCEPTION D'UNE EXPOSITION

De récentes recherches ont mis au jour les stéréotypes et représentations de genre à l'œuvre dans les scénographies d'expositions et les supports de médiation de la culture scientifique. Cet article examine le processus de conception des expositions et leur organisation sous l'angle du genre. Il se situe dans le sillage des études sur les pratiques professionnelles dans les musées qui, en coulisses, participent à produire les espaces et contenus à disposition des publics. L'article propose un éclairage critique sur le genre au musée, à partir d'une enquête ethnographique au sein des univers de conception des expositions. Cette recherche porte sur la production d'une figure de visiteuse lors de la conception d'une exposition sur le jeu vidéo, conçue par un centre de culture scientifique, technique et industrielle. Il s'agit de considérer l'exposition non seulement comme un espace matériel spécifique à vocation publique, mais aussi comme un projet autour duquel une diversité de personnes se réunissent. Cela implique d'observer l'ensemble des participants et des participantes à sa conception, leurs interactions, leur coopération mais aussi leurs résistances.

Mots clés : genre, conception, technologies, usages, jeux vidéo

\section{Abstract \\ IMAGINING WOMEN VISITORS. GENDER SCRIPTS AND THE DESIGN OF AN EXHIBITION.}

Recent studies has shown gendered stereotypes and representations at work in exhibitions about scientific culture, in their scenography or their medias. This article examines the process of exhibit design and its organization from a gender perspective. It is situated in the wake of studies on professional practices in museums which, behind the scenes, help to produce the spaces and contents available to the public. The article provides a critical perspective on gender in the museum, from a fieldwork on the design of an exhibition. This research focuses on the production of a feminine visitor figure during the design of a video game exhibition, conceived by a center for scientific, technical and industrial culture. It considers the exhibition not only as a specific material space for a public purpose, but also as a project around which a diversity of actors come together. This implies observing all the participants in its design, their interactions, their cooperation but also their resistance.

Key words : gender, design, technologies, uses, video games 
De récentes recherches ont mis au jour les stéréotypes et représentations de genre à l'œuvre dans les scénographies d'expositions et les supports de médiation de la culture scientifique, technique et industrielle (Bernardis, 2013 ; Détrez \& Piluso, 2014 ; Machin, 2010). Ces études soulignent l'absence des femmes dans les mises en scène des sciences et de leur histoire, ainsi que leurs statuts subalternes d'élèves ou d'assistantes, et le rôle maternel qui leur est souvent attribué. Nous proposons ici d'examiner le processus de conception des expositions et leur organisation sous l'angle du genre. Il s'agit donc d'ouvrir la « boîte noire » que constituent souvent les expositions (Macdonald, 1998 : 2), dont les processus de production demeurent relativement invisibles, tant aux yeux des publics qu'à ceux des chercheurs et des chercheuses. Cette recherche se situe dans le sillage des études sur les pratiques professionnelles dans les musées au quotidien qui, en coulisses, participent à produire les espaces et contenus à disposition des visiteurs et visiteuses (Beltrame, 2012; Coville, Couillard \& Schlageter, 2016 ; Macdonald, 2002).

Comment une enquête ethnographique peut-elle apporter un éclairage critique sur le genre au musée, au sein même des univers de conception des expositions ? Pour répondre à cette question, nous nous intéresserons à la production d'une figure de visiteuse lors de la conception des dispositifs de médiation de l'exposition Video Games Culture. Conçue par un centre de culture scientifique, technique et industrielle, cette exposition porte sur la culture, la pratique et l'industrie du jeu vidéo en Europe. Nous examinerons les représentations de genre que l'usage de cette figure féminine sous-tend et produit. Ce questionnement nous amène à considérer l'exposition non seulement comme un espace matériel spécifique à vocation publique, mais aussi comme un projet autour duquel une diversité de personnes se réunissent. Cela implique d'observer l'ensemble des participants et participantes ${ }^{1}$ à sa conception, leurs interactions, leur coopération mais aussi leurs résistances.

\section{SCRIPT DE GENRE ET CONCEPTION MUSEOGRAPHIQUE}

Le développement des études des publics permet une connaissance fine des visiteurs et visiteuses de musée. Les enquêtes préalables et les évaluations formatives visent à réduire l'incertitude que représente le futur public de l'exposition (Triquet \& Davallon, 1993 : 72). En dehors de ces processus encadrés, les chercheurs et chercheuses s'intéressant à la représentation des usagers et des usagères au cours de la conception des techniques ont noté que le travail de conception se compose de petites décisions quotidiennes qui façonnent le fonctionnement technique et symbolique des objets produits. En l'absence d'un public qu'il serait possible de convoquer à tout moment, les muséographes s'appuient au jour le jour sur des représentations informelles et abstraites de visiteurs et de visiteuses ou se projettent en train d'utiliser l'objet ${ }^{2}$ pour nourrir et argumenter leurs partis pris.

Les approches féministes de la sociologie des sciences et techniques poursuivent ces perspectives et s'intéressent au script de genre produit par ces pratiques (Cockburn, 1997 ; Oudshoorn et al., 2004, Van Oost 2003). Pour produire des objets, concepteurs et conceptrices imaginent et anticipent les compétences, les motivations et les comportements des futurs usagers et usagères. Dans le même temps, ces caractéristiques sont associées, de manière plus ou moins explicite, à des identités de genre et à des rôles sociaux (celui de mère, par exemple). Le script est la mise en forme technique, par les concepteurs et les conceptrices, de ce scénario d'usage imaginé - et les représentations genrées qui le sous-tendent - qu'ils inscrivent dans le fonctionnement et la matérialité de l'objet conçu. Dans le cas des expositions, ces représentations genrées nourrissent les choix de conception, et façonnent les installations interactives, tout comme l'agencement de l'espace ou encore la sélection des contenus. Si ce script ne détermine pas les modalités de visite, il contribue largement aux possibilités d'action des publics, car il privilégie certaines actions et en contraint d'autres. 
Dans Video Games Culture, l'analyse des processus de conception révèle la coexistence de deux figures de publics, l'une énoncée au masculin, l'autre au féminin. Elles correspondent à deux postures de visite, la première plutôt basée sur l'action et l'interaction avec les installations interactives, la seconde fondée davantage sur l'observation et la lecture, et caractérisée par une sorte de crainte à l'égard des technologies. Dans cette exposition, qui favorise les activités collectives, les muséographes attendent un public familial et conjugal, hétérosexuel, dont les modalités de visite sont parfois envisagées selon une logique binaire et genrée. Ainsi, loin d'être opposées, ces deux figures semblent complémentaires, et représentent deux personnes d'un même groupe de visiteurs et de visiteuses. Ce script participe donc autant à organiser les relations entre les individus et les objets de l'exposition que les relations entre les individus eux-mêmes, et suggère une répartition genrée des activités et des compétences au sein des groupes.

Souvent perçues comme abstraites, ces figures jouent pourtant un rôle très concret dans la matérialité et le fonctionnement des dispositifs de médiation, mais aussi dans la production de l'exposition. Pour étudier les interactions entre ces figures de visiteurs et de visiteuses et l'organisation sociale de la conception de l'exposition, nous nous appuyons sur des matériaux ethnographiques collectés dans le cadre d'une enquête de terrain. La première phase de cinq mois a consisté en une observation de la conception de l'exposition Video Games Culture, qui mobilise des objets techniques issus des jeux vidéo. En complément, nous avons mené quinze entretiens avec des membres de l'équipe et analysé des documents de conception ${ }^{3}$. Au regard des données collectées, il nous a semblé important d'ancrer cette réflexion dans le champ des approches féministes de la sociologie des sciences et techniques, qui étudie la construction mutuelle du genre et de la technique, et considèrent genre et technique comme deux processus sociaux enchevêtrés et constamment négociés, qui se font et se transforment dans les interactions entre les individus et avec les objets (Chabaud-Rychter \& Gardey, 2002; Cockburn \& Ormrod, 1993). Aussi, dans cet article, nous nous interrogerons tout d'abord sur la prise en compte du genre dans les politiques d'égalité de l'institution, afin de comprendre le champ d'action qu'elles définissent et leur réception au sein des équipes de conception. Nous analyserons ensuite la construction d'une figure de visiteuse, qui contribue à associer les femmes à une incompétence technique, et à une posture d'accompagnatrice ou de lectrice. Enfin, nous verrons que cette représentation participe tout aussi largement à l'organisation sociale de la conception, en particulier à l'attribution, ou non, d'une expertise entre les différents membres de l'équipe muséale.

\section{LA RÉCEPTION DU GENRE ET DES POLITIQUES D’ÉGALITÉ}

Dans In the Way of Women, Cynthia Cockburn (1991) étudie la manière dont les cultures technologiques et managériales des entreprises et de leurs dirigeants façonnent les conditions de travail des femmes et des hommes. Au sujet des politiques d'égalité et de parité, la chercheuse montre dans cet ouvrage que leur définition des objets privilégiés de l'égalité circonscrit la place des femmes dans l'entreprise et leur délègue un certain nombre de responsabilités. Elle montre, d'autre part, que les salariés perçoivent généralement ces politiques comme une contrainte voire une menace, et développent des stratégies d'évitement et de résistance pour se maintenir en dehors du champ d'action de ces politiques.

$\mathrm{Au}$ moment où Video Games Culture est conçue, l'institution muséale met en place de nouvelles politiques d'égalité. Elle s'appuie pour cela sur une évaluation de ses expositions au prisme du genre, commandée à un groupe d'expertes et d'experts afin d'élaborer par la suite des politiques d'action en faveur de la parité. Tout cela participe à définir les responsables et les champs d'action de ces politiques. Au cours des ateliers mis en place en interne pour restituer cette évaluation et travailler à une charte pour l'égalité dans l'offre muséographique de l'institution, on constate que la thématique des rapports sociaux et des inégalités de genre se réfère surtout à l'inclusion des femmes. Les responsables des ateliers soulignent l'absence 
des femmes dans le domaine des sciences et techniques, et les actions envisagées portent donc spécifiquement sur cette population ignorée. Par exemple, certaines des solutions évoquées visent à agrémenter les objets techniques pour les présenter autrement : " soigner la déco, le graphisme », «mettre de la couleur», « diffuser de la musique ». Bien que « les blagues misogynes » et « les regards insistants » soient soulignés comme des freins à la présence des femmes dans le domaine des sciences et techniques, les solutions discutées pour créer un contexte qui leur serait favorable tendent à considérer leur absence comme le résultat d'un désintérêt personnel et individuel, un manque de "goût» ou une crainte pour les technologies.

Pour lutter contre les stéréotypes de genre dans les expositions, l'institution souhaite élaborer un cahier des charges de l'égalité, renommé " charte " ou "recommandations ", afin de ne pas évoquer une "contrainte ». Ces recommandations se restreignent en grande partie aux contenus visuels, textuels et audiovisuels des expositions. La lutte contre les stéréotypes serait possible en modifiant les pratiques de rédaction et de sélection de contenus et d'illustrations. Mais il apparaît que les installations interactives, leur fonctionnement ou encore leur cadre d'usage demeurent peu interrogés durant ces ateliers. Cela conduit également les équipes à définir les muséographes comme les principaux responsables de ces politiques, lorsqu'il s'agit du domaine des expositions. D'autres identités professionnelles, liées à la conception technique et multimédia par exemple, sont moins valorisées. Cette répartition attribue aux muséographes la production du contenu des expositions, pouvant être sujet aux stéréotypes, tandis que d'autres identités professionnelles viendraient en renfort (Becker, 1988 :98) pour assurer la conception des supports de ces contenus.

Au cours de la conception de Video Games Culture, la thématique de l'égalité a été largement débattue et une partie de l'équipe ${ }^{4}$ s'est interrogée sur la nécessité de l'évoquer, en créant un dispositif de médiation dédié aux problématiques de genre dans les jeux vidéo. Pourtant, cette hypothèse a vite été éludée par les responsables du projet d'exposition. L'un d'eux nous explique cette décision lors d'un entretien :

On s'est posé [la question] dès le début d'une certaine manière en disant : c'est pour tout le monde. En gros on se l'est posée sans se la poser, en disant : voilà, c'est pour tout le monde. Il y a autant d'hommes que de femmes qui jouent donc on ne va pas s'embarrasser avec ces histoires-là.

L'analyse des entretiens et l'observation des réunions montrent que la prise en compte du genre est perçue comme une contrainte. Alors que les femmes muséographes insistent pour inclure cette perspective de genre dans leur propos, le responsable ajoute : « moi, je ne voulais pas non plus que ça tombe dans quelque chose d'un peu... trop militant ». Sur le même sujet, un autre responsable du projet estime que la prise en compte du genre aurait conduit à présenter «des jeux vidéo pour les filles» et à créer un clivage entre les publics. La considération d'un groupe social spécifique serait perçue comme "militante » et clivante. Ainsi, le second responsable explique :

En disant: "on ne fait rien sur la question", au moins, on n'interdit rien à personne, on le fait accéder à tous. Pour nous, c'est ouvert à tous, garçons et filles, handicapés, etc.

Une réflexion sur le genre se trouve donc évacuée au profit du postulat universaliste que l'exposition s'adresse «à tout le monde ». On retrouve ici les constats de Wayne Breckus (2005) sur le marquage social. Selon le sociologue, certaines catégories de l'identité apparaissent " non marquées », c'est-à-dire qu'elles demeurent non remarquées, invisibles et, par là même, considérées comme universelles - comme dans le cas de l'identité masculine. Les catégories «marquées » définissent, en creux, l'autre, le différent, le singulier. Le non marqué dépend donc du marqué pour construire son statut de catégorie par défaut, comme une absence de différence : les individus non marqués sont considérés comme n'ayant pas de spécificités liées à un groupe social. Pour le genre, cette catégorisation repose sur un modèle binaire de la perception sociale où les femmes seraient marquées (genrées), tandis que les hommes demeureraient du côté du neutre et de l'invisible. Dans ce cas de figure, la prise en 
compte des femmes insinuerait un risque de clivage : concevoir une exposition pour « tout le monde » ne serait pas compatible avec la prise en compte délibérée de différents groupes sociaux.

Dans ce silence général face au genre, les femmes muséographes œuvrent à sa prise en compte par la rédaction de deux courts textes sur la présence des femmes dans les jeux vidéo. Ceux-ci viennent compléter deux textes, déjà rédigés par les commissaires scientifiques pour être présentés sur les cimaises de l'exposition. Par exemple, à la suite d'un texte accompagnant un dispositif dédié aux personnages de jeux vidéo, le texte suivant est rédigé :

Les personnages relèvent la plupart du temps des stéréotypes : des hommes musclés prêts à l'action, des femmes aux formes caricaturales destinées à satisfaire le regard masculin. Alors qu'il offre la possibilité de jouer avec les genres et de s'essayer à d'autres identités, le jeu vidéo se contente encore souvent de reconduire les clichés sociétaux.

Les débats sur la production de ces textes soulignent le rôle de ces femmes. Lors de discussions internes entre les femmes muséographes et le chef du projet, celles-ci pensent obtenir son accord pour rédiger un texte sur le genre. Une réunion d'équipe prend place et le chef de projet passe en revue l'avancée de chaque dispositif de l'exposition pour valider avec les commissaires scientifiques les orientations choisies. L'une des muséographes se rappelle : « Toute la réunion s'est passée. Arrive la fin, ça n'avait pas été abordé. Je regarde [un chef de projet] qui me regarde du genre : "ben vas-y" ». Elle interprète ce silence comme le signe que, le genre, «c'est [elle] que ça concerne, [et qu'eux] ça ne [les] concerne pas ». Alors qu'elle vient d'arriver sur le projet pour prêter main forte - il s'agit de sa première réunion avec les commissaires scientifiques (« je ne les connaissais pas. Je débarquais ») -, elle prend malgré tout la parole à la fin de la réunion, au moment où les commissaires rangent leurs affaires : "l'exposition ne parle absolument pas du genre », lance-t-elle. Dans un premier temps, ces derniers sont dubitatifs. L'un d'eux estime que le genre est un sujet parmi d'autres et que l'exposition ne peut pas traiter de tous les sujets. L'autre craint qu'un texte traitant des équipes de conception largement masculines risque de «perpétuer les stéréotypes ». Les deux femmes de l'équipe parviennent néanmoins à faire valoir leur point de vue et finissent par obtenir la responsabilité de traiter du genre.

Tout au long de la conception de l'exposition, on observe l'équipe se coordonner pour produire des éléments cohérents, de façon à donner l'impression qu'une voix unique s'exprime. Une partie de l'équipe se détache de la rédaction des textes sur le genre : ils sont présentés comme l'initiative personnelle des femmes, que les responsables auraient "laissées $s$ 'exprimer ». En retour, elles mettent en place un réseau de coopération parallèle composé majoritairement de femmes à qui a été largement délégué le travail rédactionnel. Elles s'assurent ainsi bien de la prise en compte du genre dans l'exposition, et recrutent d'autres femmes dont les expertises se complètent (experte du comité scientifique, traductrice, correctrice, etc.) afin d'assurer la rédaction, la validation, la relecture et l'intégration de ces textes dans l'expositio.

Pour résumer, si le genre est pris en compte dans les images et les discours, il ne l'est pas dans les installations interactives, leur fonctionnement et le contexte de leur usage. Il se réduit à l'inclusion des femmes et se trouve aussi délégué aux femmes. Les modifications de l'exposition pour valoriser le genre ont lieu à la marge, font l'objet de débats, et mobilisent des réseaux de coopération différents pour assurer leur existence. La perception du genre comme une préoccupation exclusivement féminine participe à définir cette approche comme militante et contraignante par les membres qui ne s'estiment pas concernés, renforçant par là même l'invisibilité de leur rôle dans ces processus. Ceux-ci estiment au contraire que la prise en compte de groupes sociaux minoritaires œuvre contre la production d'une exposition « pour tout le monde ». Pourtant, si une représentation explicite des femmes est récusée, les représentations des usagers et usagères néophytes participent largement à présupposer une 
identité féminine.

\section{UN PUBLIC NÉOPHYTE AU FÉMININ}

Dans le contexte des installations interactives produites par les musées, les visiteurs et les visiteuses sont également des usagers et des usagères. Ils et elles tentent de comprendre les significations privilégiées par l'institution ainsi que ses attentes envers les publics. Pour agir et donner du sens aux installations, les publics cherchent les traces des significations et du mode d'adresse encodés par les muséographes. Dans cette relation, le concepteur « est obligé d'anticiper à chaque étape la réaction du visiteur, et le visiteur est obligé à chaque étape de se demander ce qu'on attend de lui. La machine est le médiateur de ce dialogue serré » (Le Marec, 1993 :101-102). Cet ajustement réciproque se déroule, dès la conception, au moment même où les muséographes imaginent, parfois de manière informelle, des figures de visiteurs et de visiteuses aux motivations et compétences variées. Ces représentations nourrissent les choix de design et façonnent la matérialité et le fonctionnement des installations.

Les responsables de Video Games Culture souhaitent concevoir une exposition « pour tout le monde ", ce qui les amène à mobiliser la figure d'un « visiteur néophyte ». Or, cette figure est convoquée de manière récurrente par des membres de l'équipe, sous les traits d'une femme, tantôt «mère de famille ", tantôt " compagne », préférant observer son compagnon plus à l'aise avec les technologies numériques. Dans sa version la plus caricaturale et dévalorisante, cette figure est parfois nommée « Madame Michu ».

$\mathrm{Au}$ cours de l'enquête, nous avons relevé les caractéristiques associées à cette figure de visiteuse pour en brosser le portrait, à partir d'entretiens et de l'analyse de documents de conception, en particulier d'une typologie de publics cibles conçue dès les prémices du projet. Les profils masculins ont en commun d'être des amateurs de jeux vidéo. Ils sont « joueurs » et se rendraient dans l'exposition pour leur propre plaisir ou par intérêt professionnel. Au contraire, la "mère de famille » incarne l'accompagnatrice d'un autre individu, conjoint ou enfants : elle est caractérisée par son identité statutaire de mère ou de compagne. Elle serait motivée par son souci de la bonne éducation, son rôle parental et ses missions d'éducation. Sa démarche serait, par ailleurs, motivée par une inquiétude à l'égard des technologies numériques, qui résulterait d'une absence de connaissance et de pratique. Par exemple, dans la typologie esquissée, chaque type de public est associé à un objet technique ${ }^{5}$ - ordinateur, smartphone ou console portable - sauf dans son cas. Elle est en effet caractérisée par son nonusage des technologies numériques. Or, pour l'équipe, expertise et usage iraient de pair, et l'absence d'usage implique une absence de connaissances dans le domaine du jeu vidéo.

La figure du ou de la néophyte sert de support d'argumentation lors de la conception de l'exposition. Le public potentiel d'une exposition, « inconnue inhérente au processus de production », constitue pour les muséographes un "outil stratégique » pour maitriser plus généralement les processus de production des contenus et des modes de communication (Triquet \& Davallon, $1993: 68 ; 72$ ). En tant que contrainte, la figure du public confère un certain pouvoir à celui ou à celle qui la convoque au cours des négociations. Sharon Macdonald qualifie de visitor hijacking cette stratégie rhétorique qui permet d'appuyer une proposition muséographique ou de la rejeter (Macdonald, 2002 : 80). La représentation d'un public imaginé est, de plus, rarement positive. Pour justifier le changement de certains éléments de l'exposition, la mise en scène d'une personne qui ne comprend pas, ou qui adopte un comportement absurde, est souvent mobilisée et, sur notre terrain, associée fréquemment aux femmes.

Cette représentation permet notamment de discuter le ton et les mots employés dans les paratextes de l'exposition. Pour argumenter ces choix, les muséographes présentent cette figure néophyte comme une visiteuse modèle et concernée, dès qu'il s'agit d'éléments de l'exposition à lire ou à regarder : elle lit, se documente et cherche des informations, en raison 
de ses inquiétudes et de ses enjeux éducatifs liés à son rôle de mère. Ainsi, même lorsqu'il est question des objets techniques de l'exposition, les éléments discutés lorsque cette figure est évoquée demeurent des éléments textuels ou discursifs : le message qu'un dispositif est censé véhiculer, la clarté d'une consigne écrite ou orale, l'exactitude d'un texte accompagnant une illustration. L'activation de la figure d'un visiteur néophyte, souvent pensé au féminin, permet donc avant tout de moduler les textes, et non de proposer un nouveau regard sur les objets techniques eux-mêmes. Les visiteuses néophytes évoquées sont censées lire les textes avant d'être usagères des installations interactives.

D'une part, la construction de cette figure de visiteuse renvoie les femmes à des « réceptrices craintives et passives » des technologies (Dagiral, 2006 :197), qui seraient incompétentes techniquement, ne pratiquant pas les jeux vidéo, et ignorantes de ce domaine. D'autre part, caractériser cette figure par son absence d'usage des technologies numériques amène l'équipe à considérer les éléments textuels de l'exposition lorsqu'elle cherche à mobiliser cette figure néophyte. Au fil de la conception, l'équipe vient associer «la » visiteuse à une posture d'observatrice et de lectrice, mais non d'utilisatrice régulière des installations interactives.

\section{LE RÔLE ORGANISATIONNEL DES VISITEUSES}

Des premiers rapports de Reine Prat $(2006,2009)$ à la récente enquête du DEPS sur La Lente Féminisation des professions culturelles (Patureau, Volat, \& Gouyon, 2016), l'étude des rapports sociaux de genre et des disparités professionnelles dans la culture souligne que, si les pratiques culturelles sont majoritairement investies par les femmes, elles n'impliquent pas leur présence accrue dans les professions culturelles. De nombreuses questions se posent sur le rôle des structures et des organisations des institutions dans ces disparités. Notre enquête permet de nourrir ce questionnement par l'étude du rôle des figures de visiteurs et de visiteuses dans l'organisation même de l'équipe et des processus de conception.

Selon les concepteurs rencontrés, le terme « Madame Michu » peut décrire « tout le monde » :

Pour nous c'est un peu le tout public. C'est le public qui n'est pas forcément expert dans quelque chose. Chacun peut être la Madame Michu, de quelque chose, parce qu'il n'en est pas expert. Mais ce n'est pas forcément péjoratif.

Au contraire, la dimension genrée de cette figure excède les femmes de l'équipe : « quelqu'un qui ne s'y connaît absolument pas, c'est forcément Madame Michu [...] c'est un stéréotype. Elle n'a pas d'âge, c'est toutes les femmes. Il y a une forme de misogynie », commente l'une d'elles. Celles-ci se sentent personnellement visées par le renvoi de l'incompétence technique aux femmes, et en particulier aux mères. Lors de la conception de l'exposition, les femmes muséographes se rendent par exemple dans un festival de jeux vidéo, et nous racontent que des journalistes viennent les photographier dès qu'elles se saisissent d'une manette pour jouer : "Pour eux, c'est étonnant de voir deux mères de famille de 40 ans. On était les Madame Michu du festival », conclut l'une d'elles. Les femmes de l'équipe partagent en effet des caractéristiques communes avec cette visiteuse imaginaire (femme, mère de famille, qui s'interroge sur la pratique vidéoludique de ses enfants). Celles-ci se retrouvent à devoir composer avec ces ressemblances au quotidien dans leur travail. Ces comparaisons jouent par exemple un rôle dans le crédit qui leur est ou non accordé. Elles ont des implications très concrètes dans l'organisation de l'équipe et la division du travail, comme dans la production des dispositifs de médiation.

Les femmes muséographes s'opposent à ce stéréotype pour disputer une expertise ou, au contraire, composent avec celui-ci pour construire un parti-pris autour de cette représentation. Une muséographe se sent particulièrement visée par cette représentation caricaturale: « Madame Michu, c'est moi ! » insiste-t-elle parfois en réunion, pour lutter contre le mépris qui entoure cette figure. Elle reprend à son compte cette identité péjorative pour l'incarner et lui donner une voix. Par exemple, les chefs de projet souhaitent se démarquer de la pratique 
du jeu vidéo dans la sphère domestique, associée à une posture assise et immobile. Pour cela, ils conçoivent des installations pour mettre en scène le corps des publics. L'exposition est un " théâtre des habiletés techniques » (Dodier, 1993 :115), où il est nécessaire d'oser se mettre en scène pour interagir avec une partie des installations. Dans un entretien où la muséographe estime que «Madame Michu, c'est notre public. Enfin, ça devrait», elle revient sur ce parti pris :

Certains éléments, il faut déjà être un peu joueur pour le faire. Au-delà de la difficulté du truc, je pense qu'il y a, pour des personnes comme moi par exemple... Et encore moi, je fais des expos. Je ne suis pas le public lambda non plus. Mais des personnes qui n'ont vraiment pas l'habitude des nouveaux outils, qui ne sont pas à l'aise... Tu peux avoir du public un peu timide de se mettre en scène. C'est pas facile de se mettre en scène. Moi, en public, je ne suis pas très à l'aise pour mettre mon corps en scène. Certains éléments de l'expo, je les regarde, je les trouve formidables, j'ai dû les faire en confidence, quand il y avait des prototypes, mais dans l'expo, je ne pense pas que je vais les faire.

C'est par son inhibition face aux dispositifs conçus que la muséographe souligne l'importance des textes qui les accompagnent, tandis qu'elle attribue aux «joueurs » l'aisance nécessaire pour utiliser les installations dans l'espace public de l'exposition. Elle part de son expérience pour réhabiliter ce public jugé néophyte, parfois non-usager, afin d'insister sur l'accessibilité des dispositifs et la qualité des explications pour inclure ces publics, ne pas les délaisser au profit de dispositifs spectaculaires. Cependant, par ces comparaisons, elle conforte parfois ses collègues dans l'association entre une figure féminine et l'inhibition à l'égard des technologies.

En effet, si cette figure de visiteuse façonne les contenus de l'exposition et les postures de visite privilégiées, elle joue aussi un rôle dans la répartition des tâches et des compétences entre les membres de l'équipe au cours de la conception, et en particulier dans le crédit et le pouvoir décisionnel accordés ou non à chacun·e. Dans l'étude ethnographique qu'il fait d'une équipe de conception de jeux vidéo, Robin Johnson (2013) montre par exemple que les salariés bénéficient de prime abord d'une identité de « joueurs » et qu'on leur attribue d'office des compétences techniques et des connaissances en matière de jeu vidéo. Les femmes sont au contraire considérées en premier lieu comme «non-joueuses » et incompétentes, et cherchent à faire leurs preuves pour tenter de modifier cette représentation tenace. Dans notre enquête, nous remarquons que, lorsque des personnes partagent des caractéristiques communes avec cette figure de visiteuse, leurs connaissances, notamment techniques, peuvent être mises en doute. L'attitude d'un concepteur illustre bien cette situation. Celui-ci explique régulièrement à l'une des femmes muséographes les principes techniques du domaine dans lequel il travaille, à l'appui d'un vocabulaire très simplifié. Or, comme celle-ci le lui rappelle à chaque fois, elle se trouve être une experte de ce domaine, sur lequel elle a d'ailleurs mené une recherche doctorale.

La conception de Video Games Culture est un lieu de définition et de revendication de domaines d'expertise. Or, celle-ci porte sur un domaine - le jeu vidéo - où les savoirs sont peu stabilisés : un nombre important de membres de l'équipe revendique une expertise sur les contenus ou les technologies mises en valeur. Au jour le jour, un concepteur est considéré comme "l'expert» du jeu vidéo. Une muséographe et une médiatrice mettent parfois en doute ses préconisations et mobilisent pour cela leurs propres connaissances. Celui-ci riposte par des questions précises (la date de production d'un jeu, par exemple) pour tenter de les mettre en défaut et souligner l'étendue de ses connaissances afin de conserver ce statut. Le débat trouve généralement une issue par la division des activités : le concepteur exprime son point de vue dans la réalisation de l'installation interactive, tandis que les deux femmes s'occupent des dispositifs d'accompagnement (paratextes ou animation scientifique). Ces distinctions s'opèrent de manière concrète à travers les espaces de conception, dont l'accès dépend des compétences attribuées aux un·es et aux autres. Les éléments d'une même installation interactive (contenus, paratexte, mobilier, dispositif technique) sont alors produits 
en des lieux différents, par des individus qui ne partagent pas toujours le même point de vue.

L'expertise technique, tout comme les connaissances plus générales sur le jeu vidéo, ne sont généralement pas attribuées aux femmes muséographes, ce qui, dans certaines situations, participe à les maintenir en dehors de la conception technique des installations interactives. Pourtant ces professionnelles assurent la prise en compte du genre et, plus généralement l'accessibilité des dispositifs aux publics néophytes. Dans les espaces de conception technique des dispositifs de médiation, on observe une moindre présence de la figure néophyte, au profit d'une figure de visiteur, souvent pensée au masculin, caractérisé par des habiletés techniques, une familiarité avec les technologies numériques utilisées dans l'exposition et par une posture de visite basée sur l'usage des installations interactives.

\section{CONCLUSION : PRENDRE EN COMPTE LE GENRE AU COURS DE LA CONCEPTION}

Deux figures implicites d'usagers cohabitent au cours de la conception. Les textes sont associés à un usager - notamment une usagère - néophyte, et les objets interactifs à un usager aux compétences techniques plus importantes. Or, ces éléments se retrouvent au sein des mêmes installations, composées d'un ou de plusieurs objets interactifs, ainsi que des textes qui les accompagnent. Ces derniers ne décrivent pas l'usage, comme dans le cas d'un manuel, mais proposent une façon alternative ou complémentaire de donner du sens à l'usage de l'installation. Deux postures d'usage semblent s'inscrire dans l'exposition: une posture active, qui repose sur l'usage d'objets techniques et la mise en scène d'habiletés et du corps qui interagit, et une posture observatrice ou spectatrice, qui consiste en la lecture d'un texte, complétée par l'observation d'autres personnes en action.

Ces scripts induisent des rapports spécifiques entre identités de genre et technologies. D'un côté, l'emploi des termes «le visiteur», voire «le joueur», l'association du domaine de la technique au masculin et l'usage fréquent de la i-methodology dans une équipe de conception souvent masculine associent les compétences techniques à une identité masculine. Celles-ci se retrouvent ensuite mises en valeur par les partis pris des muséographes et des commissaires, qui ne souhaitent pas reproduire des pratiques domestiques ou privées, et leur préfèrent des installations spectaculaires et innovantes, parfois au détriment de la simplicité d'usage et d'un apprentissage relativement encadré et accompagné. D'un autre côté, l'usager néophyte est souvent associé aux femmes, par un recours aux figures de la " mère de famille » inquiète ou d'une personne « préférant regarder son compagnon ». Ces deux figures, qui reposent sur des usages et des rôles sociaux différents, se construisent mutuellement à travers la représentation d'une visite en famille ou en couple, hétérosexuel, où les motivations et les rôles des visiteurs et des visiteuses imaginés sont répartis selon les identités de genre. L'enquête des publics effectuée souligne bien évidemment que la relation entre scripts et usages effectifs ne peut être réduite à un «miroir » où les modalités de visite correspondent en tout point aux usages imaginés. Cependant, parmi la variété des pratiques de visite, certains usages et non-usages semblent marqués par la manière dont l'exposition et ses objets favorisent ou non l'action des publics $^{6}$

L'approche ethnographique des espaces de conception muséographique offre un éclairage supplémentaire pour saisir le paradoxe entre un apparent consensus sur l'égalité de droits, et la persistance, dans les faits, des inégalités de genre. L'enquête souligne que la prise en compte de ces inégalités se heurte à une conception universaliste des expositions, qui s'offriraient à «tout le monde» dès lors qu'elles sont postulées comme telles. Ce postulat demeure aveugle tant aux inégalités qui préexistent à l'exposition qu'à celles qui traversent et façonnent sa conception. L'enquête invite à articuler la production de figures de visiteurs et de visiteuses, l'organisation sociale de la conception, la division des activités et l'attribution ou non d'une expertise, l'accès aux activités et aux espaces professionnels, ou encore la production des dispositifs de l'exposition, afin de "suivre» les rapports sociaux de genre 
dans les différents lieux de la conception muséographique. En d'autres termes, l'observation au long cours invite à décentrer notre regard des contenus muséographiques présentés aux publics, pour l'étendre aux coulisses et considérer les pratiques quotidiennes, matérielles et routinières des hommes et des femmes qui les produisent.

\section{BIBLIOGRAPHIE}

Akrich (Madeleine). 1987. « Comment décrire les objets techniques? », Techniques et culture (9), p. 49-64.

Akrich (Madeleine). 1995. «User representations : Practices, Methods and Sociology », p. 167-184 in Managing Technology in Society / sous la direction de Rip (Arie), Misa (Thomas) et Schot (Johan). Londres : Pinter.

Becker (Howard). 1988. Les mondes de l'art. Paris : Flammarion.

Beltrame (Tiziana). 2012. Ethnographie de la patrimonialisation : numériser, inventorier et classer la collection du musée du quai Branly. Thèse de doctorat en Anthropologie. Université Paris 10.

Bernardis, (Marie-Agnès) 2013. « La culture scientifique, une culture au masculin ? ». La Lettre de l'OCIM, (149), p.16-24.

Brekhus (Wayne). 2005. « Une sociologie de l'« invisibilité » : réorienter notre regard ». Réseaux, (129-130), p.243-272.

Chabaud-Rychter (Danielle), \& Gardey (Delphine). 2002. L'engendrement des choses. Des hommes, des femmes et des techniques. Paris : Archives contemporaines.

Cockburn (Cynthia). 1991. In the way of women : Men's resistance to sex equality in organizations. Londres : Macmillan.

1997. « Domestic technologies : Cinderella and the engineers ». Women's Studies International Forum, 20, p. 361-371.

Cockburn (Cynthia) \& Ormrod (Susan). 1993. Gender and Technology in the Making. Londres : SAGE.

Coville (Marion), Couillard (Noémie) \& Schlageter (Karin). 2016. « Entrée réservée au personnel ». Poli-Politique de L'image, (12, Les coulisses du musée), p. 6 - 12.

Dagiral (Eric). 2006. « Genre et technologie ». Terrains et Travaux, 1(10), p. $194-206$.

Détrez (Christine), \& Piluso (Claire). 2014. « La culture scientifique, une culture au masculin ? » p.27-51 in Questions de genre, questions de culture / sous la direction de Sylvie Octobre, Paris : La documentation française.

Dodier (Nicolas). 1993. «Les arènes des habiletés techniques ». Raisons Pratiques, (4), p.115-139.

Johnson (Robin). 2013. « Toward Greater Production Diversity Examining Social Boundaries at a Video Game Studio ». Games and Culture, 8(3), p.136-160.

Le Marec (Joëlle). 1993. « L'interactivité, rencontre entre visiteurs et concepteurs ». Publics et Musées, (3), p.91-109.

Macdonald (Sharon). 1998. The politics of display : Museums, science, culture. Londres : Psychology Press.

2002. Behind the Scenes at the Science Museum. Londres : Bloomsbury. 
Machin (Rebecca). 2008. «Gender Representation in the Natural History Galleries at the Manchester Museum », Museum \& Society, 6/2008, p. 54-67.

Oudshoorn (Nelly), Rommes (Els), \& Stienstra (Marcelle). 2004. « Configuring the User as Everybody : Gender and Design Cultures in Information and Communication Technologies ». Science, Technology \& Human Values, 29(1), p.30-63.

Patureau (Frédérique), Volat (Gwendoline), \& Gouyon (Marie). 2016. « La lente féminisation des professions culturelles ». Culture Études, 2016-2.

Prat (Reine). 2006. Mission pour l'égalité et contre les exclusions, rapport d'étape $n^{\circ} 1$. Paris : Ministère de la culture et de la communication.

Prat (Reine). 2009. Mission pour l'égalité hommes / femmes, rapport d'étape $n^{\circ} 2:$ De l'interdit à l'empêchement. Paris : Ministère de la culture et de la communication.

Triquet (Eric) \& Davallon (Jean). 1993. « Le public, enjeu stratégique entre scientifiques et concepteurs », Publics et Musées, (3), p. 67-90.

Van Oost (Ellen) 2003. « Materialized gender : how shavers configure users' femininity and masculinity », p.193-208 in How Users Matter : The Co-construction of Users and Technology / sous la direction de Nelly Oudshoorn \& Trevor Pinch. Cambridge : MIT Press. 
La rédaction d'un article sur les musées au prisme du genre suppose une réflexion sur l'écriture scientifique et la visibilité qu'elle donne aux hommes et aux femmes. Le point médian est souvent d'usage pour conserver la concision du texte (visiteur·ses). Afin de respecter l'harmonie et la politique éditoriale de la revue, celui-ci a été ici remplacé par un recours systématique aux formes féminines et masculines dans toute leur intégrité (visiteurs et visiteuses).

\section{2}

Ce procédé est notamment identifié et étudié par Madeleine Akrich, qu’elle propose de nommer « i-methodology » (Akrich, 1995).

3

Une seconde phase, d'une durée de 10 mois, a consisté en une étude des publics de l'exposition, de leur parcours, de leurs usages des dispositifs de médiation, et du sens qu'ils et elles donnent à cette visite.

4

Le genre est d'autant plus central qu'au même moment, une controverse internationale émerge autour du sexisme et de la place des femmes dans le domaine du jeu vidéo, en raison du harcèlement que subissent des joueuses, des conceptrices et des journalistes.

5

Une seconde visiteuse est représentée, la « joueuse casual », liée à une pratique nommée « jeu occasionnel », terme souvent employé pour se référer à des supports matériels (tablettes, smartphones) ainsi qu'à des types de jeux (logique, puzzle, comme Candy Crush). Le terme « casual » définit donc bien souvent des jeux et leurs publics, plus qu'une fréquence de pratique. Il est important de noter d'une part que l'on refuse parfois à ces productions l'appellation « jeu vidéo » (l'argument récurrent est que ces jeux ne supposeraient ni challenge ni compétence) et d'autre part, que ces pratiques sont souvent présentées comme féminines.

6

Voir Coville M., « Des experts et des non-initiées ? Rôles de visiteur·ses au cours de la médiation et de la visite d'une exposition scientifique sur le jeu vidéo », Sexe et genre de la culture : production, médiation et consommation, DEPS-ministère de la Culture / ENS Lyon (à paraître). 